\title{
Average size perception and the allure of a new mechanism
}

\author{
DANiEL J. Simons \\ University of Illinois at Urbana-Champaign, Urbana, Illinois \\ AND \\ KRISTOFFER MYCZEK \\ University of California, Irvine, California
}

\begin{abstract}
In this brief response to commentaries by Ariely (2008) and Chong, Joo, Emmanouil, and Treisman (2008) on our earlier article, we highlight the two key assumptions underlying earlier claims about statistical summary representations of object size and argue that existing studies have not met either of them. We note why statistical summary representations of size are different from such representations of motion or orientation, and we emphasize the need for simulations of performance to exclude focused attention explanations for judgments of average size.
\end{abstract}

The claim that people perceive the average size of an array of circles in parallel challenges the long-held view that processing of the intrinsic properties of objects depends on focused attention. Strong support for a new average size perception process requires experiments that convincingly exclude more mundane explanations that are based on known mechanisms and consistent with existing theory. Our article (Myczek \& Simons, 2008) challenged the assumed need for a new mechanism by showing that performance levels found in all the published studies of average size perception also could be achieved with strategic use of focused attention.

Note that our claims were specific to the idea that people perceive a statistical summary representation of object size, and we did not address statistical summary representations for other properties, such as spatial frequency, motion, and orientation. For those sorts of features, the extraction of a statistical summary is uncontroversial; we can perceive the preponderant direction of motion or the most common orientation in a display by pooling across receptors specific to individual motion directions or orientations, without focusing attention on individual objects. Size is not analogous to motion or orientation, though, because there are no known receptors for individual absolute sizes. Size is typically thought to be an intrinsic property of an object that is not perceived independently of the object itself, and that is what made the original claim that people perceive the average size of an array of objects in parallel interesting, provocative, and different from earlier claims about statistical summary representations.

The conclusion that people perceive the average size of a set of circles in parallel was based on two assumptions: (1) Performance on average size judgment tasks exceeded what could be accomplished within the limits of focused attention, and (2) performance must result from averaging the sizes of all the items in the display. Assumption 1 was the basis for the published claims of an average size perception mechanism, but our simulations showed the assumption to be false for all of the previously published experiments. In their response, Chong, Joo, Emmanouil, and Treisman (2008) acknowledge that "it is, in fact, quite difficult to devise tests that require samples of more than four or five items, simply because samples of increasing size quickly approximate the population mean" (p. 1329). In other words, it is difficult to devise a study that validates Assumption 1, which is consistent with our claim that no previous studies adequately did so. To validate Assumption 1, thereby supporting the need for a new mechanism, performance must exceed what could be accomplished by sampling four or five items.

Note that empirical verification of Assumption 1 does not validate Assumption 2; performance exceeding what could be accomplished from sampling four or five items with focused attention does not imply perception of the average size of all the objects in a display in parallel. It would imply some process other than (or in addition to) focused attention, but that process might not involve a true statistical summary representation of the sort thought to operate in scene gist perception. Far stronger evidence would be required to support Assumption 2, and none of the existing experiments have provided such evidence.

Our article admittedly presented a grab bag of focused attention strategies to account for the extant findings. Of course, our claim would be more palatable if a single focused attention strategy could account for all of the existing data, but we would be surprised if such a unifying

D. J. Simons, dsimons@illinois.edu 
strategy exists. More important, our intent was not to define exactly how people perform these tasks, and we did not claim that participants must use the strategies we proposed. Rather, our goal was to test the validity of Assumption 1 - to show that, in principle, average size judgments could be accomplished using focused attention strategies. Whether or not our assortment of strategies is more parsimonious than a new, parallel average size perception mechanism is debatable, of course. None of the studies postulating a global process for average size perception have provided any mechanism by which size information can be pooled in parallel across the entire set of objects (as it can for motion and orientation). In some cases, a grab bag of strategies consistent with known mechanisms is more parsimonious than a single, new process without a known mechanism.

In their response article, Chong et al. (2008) present several new experiments testing some of the focused attention strategies that we proposed. We applaud the empirical attempt to eliminate such focused attention strategies, and we hope that further experiments successfully verify Assumption 1 (since that is far more interesting than our more mundane account). Each of their new experiments challenges the specific focused attention explanations that we proposed, but the experiments still permit other, equally plausible strategies. For example, they note that if participants used the strategies we originally proposed, then by intermixing trials requiring different strategies, participants would have to switch strategies from trial to trial. Given that such task switching is implausible, evidence that intermixing had no costs would suggest that participants were not using those strategies. Chong et al. found no effect of intermixing trial types and concluded that participants must be using a global process to perceive the average size. However, the assumption that participants must switch strategies to perform the task is not justified. Participants need not use the particular strategies that we proposed. In this case, for example, they could adopt a single strategy that happens to work for all of the intermixed trial types: They could average the biggest and smallest items from each side of the display and compare those averages. If implemented perfectly, this strategy would give the right answer on every trial. More broadly, even if the study had successfully eliminated focused attention strategies, it would not automatically support the existence of a global averaging process (i.e., Assumption 2). Other experiments in their response article similarly assumed that participants must be using the strategy we proposed (e.g., searching for the biggest item) when other focused attention strategies would work as well.

In his commentary, Ariely (2008) highlights the interesting dissociation between judging the average size of an array of objects and recognizing individual elements from the array. Whether or not the average size judgment constitutes a statistical summary representation processed in parallel across an entire array, such dissociations between memory for individual display items and judgments of the average size of a display are interesting in that they show different ways that people process the same source of information. The dissocia- tion might be between two different focused attention mechanisms or strategies, though, and might not indicate the existence of a global size-averaging mechanism. For example, if people sample a subset of items to form an average, the incentive to remember the individual items after sampling is minimal. In contrast, the incentive to remember individual items is strong when trying to recognize individual items, which might lead to a different sampling strategy. Thus, evidence that people do not remember the individual items when they are trying to judge the average may be entirely consistent with a sampling explanation.

We agree with Ariely (2008) that any model purporting to account for human performance needs to take into account the sorts of errors that human observers make. Our purpose was to show that focused attention can, in principle, account for the extant results. We did not claim that people necessarily use the strategies we proposed, just that the results are consistent with focused attention explanations. As Ariely notes, it is not entirely clear how to add judgment error to these simulations. Note, though, that if such judgment error were unbiased (i.e., equally likely to lead to a bigger or a smaller estimate of the mean), it would only add noise to the estimates and would not systematically alter the simulated performance for different strategies. The one exception would be cases in which simulated performance is already perfect. In such cases, adding judgment error could only reduce simulated performance, possibly making it more similar to actual human performance.

We hope that our simulations and critique inspire further research that convincingly eliminates focused attention explanations (verifying Assumption 1), that supports a global averaging process across the entire display (verifying Assumption 2), and that delineates a plausible mechanism underlying any proposed averaging process. At a minimum, the simulation approach we adopted in our article provides a means by which to verify whether or not focused attention strategies are sufficient in principle to account for performance, and future empirical work along the lines of the experiments in Chong et al.'s (2008) response article can help to determine whether or not people actually do use such strategies.

\section{AUTHOR NOTE}

Correspondence concerning this article should be addressed to D. J. Simons, Department of Psychology, University of Illinois, 603 E. Daniel Street, Champaign, IL 61820 (e-mail: dsimons@illinois.edu).

\section{REFERENCES}

ArIELy, D. (2008). Better than average? When can we say that subsampling of items is better than statistical summary representations? Perception \& Psychophysics, 70, 1325-1326.

Chong, S. C., Joo, S. J., Emmanouil, T.-A., \& Treisman, A. (2008). Statistical processing: Not so implausible after all. Perception \& Psychophysics, 70, 1327-1334.

MyczeK, K., \& Simons, D. J. (2008). Better than average: Alternatives to statistical summary representations for rapid judgments of average size. Perception \& Psychophysics, 70, 772-788.

(Manuscript received July 21, 2008; accepted for publication July 21,2008 .) 